\title{
Enhanced connexin 43 expression following neural stem cell transplantation in a rat model of traumatic brain injury
}

\author{
Bo Yu', Haiying Ma², Li Kong ${ }^{2,3}$, Yuxiu Shi' ${ }^{2}$ Yunhui Liu
}

${ }^{1}$ Department of Neurosurgery, Shengjing Hospital, China Medical University, Shenyang, China

2Department of Histology and Embryology, Institute of Pathology and Pathophysiology, China Medical University, Shenyang, China

${ }^{3}$ Department of Histology and Embryology, Dalian Medical University, Dalian, China

Submitted: 9 December 2011

Accepted: 8 February 2012

Arch Med Sci 2013; 9, 1: 132-138

DOI: $10.5114 /$ aoms.2012.31438

Copyright @ 2013 Termedia \& Banach

\section{Abstract}

Introduction: Reestablishment of functional networks after traumatic brain injury (TBI) has been proffered as one of the goals of neural stem cell (NSC) transplantation therapeutics. Gap junctions provide essential means for direct cellular communication by transferring small molecules and ions, which may provide insights into the interplay between grafted NSCs and host cells.

Material and methods: Thirty-six adult male Wister rats were used in this study. The controlled cortical impact $(\mathrm{CCl})$ model of brain injury has been performed. Seventy-two hours after $\mathrm{CCl}$ injury, animals were randomly assigned to two groups: PBS- and NSC- transplanted group. NSCs-transplanted group received delivery of the NSCs suspension to the cortex below the injury cavity in the ipsilateral hemisphere. At 1, 2, and 4 weeks post-transplantation, we investigated the expression patterns of gap junction-associated connexin $43(C \times 43)$ in the transplant site and the border of $\mathrm{CCl}$ by immunohistochemistry, Western blot and RT-PCR.

Results: Our findings showed that $\mathrm{Cx} 43$ staining was significantly greater in the transplant site and the border of $\mathrm{CCl}$ in the NSCs-transplanted rats compared to the control rats at different time points $(p<0.01$ at 1 week, $p<0.05$ at 2 and 4 weeks). Significantly higher gene and protein expression of $C \times 43$ was found in NSCs-transplanted rats compared to the control rats in the period of 4 weeks post-transplantation $(p<0.01)$, and remained at a higher level until 2 weeks with or without NSC transplantation.

Conclusions: It is proposed that gap junction-associated Cx43 might participate in NSCs' beneficial effects via gap-junctional coupling by which grafted NSCs integrate into host neural tissue following transplantation after TBI.

Key words: neural stem cell, traumatic brain injury, transplantation, connexin43.

\section{Introduction}

Traumatic brain injury (TBI) results in immediate neuronal and glial cell loss and associated neurological deficits. Recent knowledge on neural stem cells has brought novel approaches to the use of stem cells in the treatment of some neurodegenerative disorders, as well as in the management of central neural system (CNS) injuries [1]. Reestablishment of functional networks after TBI has been proffered as one of the goals of stem

\section{Corresponding author:} Yunhui Liu PhD

Department of Neurosurgery Shengjing Hospital China Medical University No. 36 Sanhao St, Heping District Shenyang 110004, China E-mail: yunhuiliuln@163.com Yuxiu Shi PhD Department of Histology and Embryology China Medical University No. 92 Beima Road Heping District Shenyang 110001, China E-mail: yuxiushi@163.com 
cell-mediated therapeutics. Although it is becoming recognized that grafted neural steam cells (NSCs) interact with endogenous imperiled neurons in vivo, the underlying mechanisms are not clear. Our previous study showed that grafted NSCs could improve expression of synaptic protein in the core of transplantation and improve functional recovery following cell transplantation after TBI [2]. Others have attributed this rescue and protection to the release of a protective factor or specific neurotransmitters from NSCs and signals present in the injured brain via cell-cell mediated repair $[3,4]$. However, this mechanism alone is inadequate to account for the beneficial effects of NSCs.

Gap junctions provide essential means for direct cellular communication by transferring small molecules and ions, which may provide insights into the interplay between grafted NSCs and host cells. The role played by this type of direct intercellular communication during the progression of the injuries is of some debate. Far from being a simple matter of judgment, the contribution of gap junctions to cell injury is a complicated phenomenon that depends on the specific insult and network in which it operates [5]. The alterations in the gap junction activities after brain injury have been proposed to be involved in 'bystander death', which is delayed neuronal death, by allowing the spread of death messengers from injured cells to otherwise healthy neighboring cells through the gap junction [6]. However, an early and essential way in which grafted NSCs integrate functionally into the host neural circuitry and affect host cells is via gap-junctional coupling, even before (and perhaps establishing a template for) mature electrochemical synaptic communication. The exogenous NSCs also protect host neurons from death and reduce such signs of secondary injury as reactive astrogliosis. Both in vitro and in vivo, these beneficial effects of NSCs were abrogated when gap junction formation or function was suppressed [7].

Connexin proteins form hemichannels with their typical hexamer conformation, and association of two hemichannels of adjacent cells compose a functional gap junction. The expressions of some connexins in the nervous system have been shown to be influenced by neuronal injury [8]. Of these, connexin 43 (Cx43) is the most ubiquitously expressed and is predominantly found in astrocytes, activated microglia, and developing neurons. Furthermore, Cx43 appeared to be most closely associated with immature NSCs and/or non-neuronal cells [7]. Here we report that NSC transplantation significantly increased the gene and protein expression of gap junctionassociated $\mathrm{C} \times 43$ in the transplant core and border of the $\mathrm{CCl}$ injury in NSCs-transplanted rats compared to the saline control rats at 1, 2 and 4 weeks post-transplantation. It is proposed that gap junctions might participate in the iterative developmental process by which grafted NSCs integrate into host neural tissue after TBI.

\section{Material and methods}

\section{Culturing of NSCS}

All procedures were approved by the Institutional Animal Care and Use Committee of the China Medical University, Shenyang, China. Primary NSCs were isolated and cultured as previously described $[9,10]$. In brief, the forebrain tissue of embryonic 14-day-old Wistar rats (Experimental Animal Center of Dalian Medical University, China) was harvested. Accutase (Sigma, St. Louis, MO, USA) was added to the shredded tissue and incubated for 10 min at room temperature. Gentle pipetting was used to generate a single cell suspension. The suspension was centrifuged for $5 \mathrm{~min}$, resuspended with culture medium, and then seeded into $25 \mathrm{~cm}^{2}$ T-flasks at a density of $0.8 \times 10^{5}$ cells $/ \mathrm{ml}$ as a suspension. Cells were maintained at $37^{\circ} \mathrm{C}$ in a humidified atmosphere with $5 \% \mathrm{CO}_{2}$. Expansion medium (DMEM and $\mathrm{F} 12$, 1 : 1; L-glutamine $2.92 \mathrm{~g} / 100 \mathrm{ml}$, HEPES $5 \mathrm{mM}$, $\mathrm{NaHCO}_{3} 7.5 \%$, glucose $0.915 \mathrm{~g} / 100 \mathrm{ml}$, and heparin $50 \mathrm{mg} / 100 \mathrm{ml}$, all from Sigma, USA) contained $\mathrm{N}_{2}$ supplement (1\%; Gibco, USA), epidermal growth factor (EGF), $10 \mathrm{ng} / \mathrm{ml}$ and basic fibroblast growth factor (bFGF), 10 ng/ml (Invitrogen, USA). As shown previously, their pluripotent stem cell fate was confirmed [11].

\section{Surgical procedures and transplantation}

Thirty-six adult male Wister rats (weight, 200220 g, Experimental Animal Laboratory of Dalian Medical University, China) were used in this study. All procedures were approved by the Institutional Animal Care and Use Committee of the Dalian Medical University, Dalian, China. The controlled cortical impact $(\mathrm{Cl})$ model of brain injury has been described previously [2]. Briefly, on the day of surgery the animals were deeply anesthetized with sodium pentobarbital (60 mg/kg intraperitoneally) and placed in a stereotactic frame (Stoelting Co., USA). After the skull was exposed, a 3-mm craniotomy was performed over the left parietotemporal cortex between lambda and bregma, with the dura matter left intact. The $\mathrm{CCl}$ brain injury was induced by the use of a $2.0 \mathrm{~mm}$ diameter pneumatic impactor (Air-Power, Inc., High Point, NC) (tissue deformation $1 \mathrm{~mm}, 4.5 \mathrm{~m} / \mathrm{s}$ tip velocity). Seventy-two hours after $\mathrm{CCl}$ injury, animals were randomly assigned to two groups. One group received delivery of the NSC suspension at a concentration of $1 \times 10^{5}$ cells $/ \mu \mathrm{l}$ ( $3 \mu \mathrm{l}$ per animal) to the cortex below the injury cavity in the ipsilateral hemisphere: anteroposterior $3.0 \mathrm{~mm}$ from bregma, mediolateral $1.0 \mathrm{~mm}$, dorsoventral $1.1 \mathrm{~mm}(n=18)$. The second (control) group 
received an equivalent volume of phosphate-buffered saline in the same location $(n=18)$. A $10-\mu$ l Hamilton syringe was slowly advanced through the dura and cortex until the desired depth was reached. With controlled use of the Stoelting quintessential injector (Stoelting Co., USA), the cell suspension was injected over a period of $3 \mathrm{~min}(1 \mu \mathrm{l} / \mathrm{min})$. The needle was left in place for an additional $5 \mathrm{~min}$ and then withdrawn slowly.

\section{Histological analysis}

At 1, 2 and 4 weeks, animals $(n=3$ in each group at different time points) were given a lethal dose of sodium pentobarbital ( $200 \mathrm{mg} / \mathrm{kg}$ intraperitoneally) and transcardially perfused with cold phosphatebuffered saline and freshly hydrolyzed $4 \%$ paraformaldehyde (pH 7.4 in $0.1 \mathrm{~mol} / /$ phosphate buffer). Brains were harvested, postfixed overnight, cryoprotected in increasing sucrose concentrations, and then cryosectioned at a 16- $\mu \mathrm{m}$ thickness along the coronal plane for immunohistochemistry. In brief, sections were rinsed in $0.3 \%$ Triton X-100 in phosphate-buffered saline (T-PBS) and pre-incubated in $10 \%$ normal goat serum in T-PBS, rinsed with $\mathrm{PBS}$ again, immersed in $3 \% \mathrm{H}_{2} \mathrm{O}_{2}$ for 10 min, washed with PBS, blocked by normal goat serum and incubated at $4^{\circ} \mathrm{C}$ overnight with the primary antibodies rabbit anti-Cx43 (1: 500; Sigma, USA). Then, biotinylated secondary antibody and $A B C$ complex (Boster, Wuhan, China) were added in sequence and developed with DAB reaction. Images were taken with an Olympus optical microscope.

\section{Western blotting}

Another 3 animals per group were decapitated at each time point. Brain tissue encompassing the transplant site and the border of the lesion was obtained immediately and dissected into two parts on a bed of ice along the center of the injury cavity for Western blot and RT-PCR detection (described below). Western blot analysis was performed as described previously [12]. Each sample was sonicated in $0.0625 \mathrm{M}$ Tris- $\mathrm{HCl}(\mathrm{pH}$ 6.8) and then centrifuged at $12,000 \mathrm{~g}$ for $15 \mathrm{~min}$ at $4^{\circ} \mathrm{C}$. The supernatant was assayed for protein using a Bradford assay. Aliquots $(50 \mu \mathrm{g})$ of protein were boiled in denaturing sample buffer $(62.5 \mathrm{mmol} / \mathrm{l}$ Tris $(\mathrm{pH} \mathrm{6.8)}$, $2 \%$ SDS, $5 \mathrm{mmol} / \mathrm{L}$ EDTA, $10 \%$ glycerol, 0.25\% 2-mercaptoethanol, and $0.01 \%$ bromophenol blue). Brain samples were loaded onto SDS-PAGE mini-gels, electrophoresed, and transferred to nitrocellulose membranes. After transferring, membranes were blocked with $5 \%$ milk in TBST $(0.1 \%$ Tween 20 in $20 \mathrm{mM}$ Tris- $\mathrm{HCl}, \mathrm{pH} 7.4$, and $410 \mathrm{mM} \mathrm{NaCl}$ ) for $2 \mathrm{~h}$ at room temperature. Blots were incubated overnight at $4^{\circ} \mathrm{C}$ with rabbit anti-Cx43 (1:1000; Sigma, USA). Following primary antibody incubations, blots were washed three times for 5 min each with TBST, incubated for
$1 \mathrm{~h}$ with horseradish peroxidase (HRP)-linked secondary antibodies, washed four times for 10 min each with TBST, and developed with an enhanced chemiluminescence Western blotting detection system kit (ECL Plus, Amersham Biosciences, NJ, USA).

\section{RT-PCR analysis}

Total mRNA was extracted from the tissue using an RNA isolation reagent (TRIZOL, TaKaRa, Dalian, China) according to the manufacturer's instructions and treated with RNase-free DNase (TaKaRa, Dalian, China). PCR was performed using Taq Dynazyme (TaKaRa, Dalian, China) under standard conditions. $\beta$-actin was co-amplified as an internal control. The products were observed after electrophoresis on $1.2 \%$ agarose gel, and the density of each band was analyzed on the Gel Image Analysis System. The level of $\mathrm{C} \times 43$ mRNA was determined by calculating the density ratio of $C \times 43 \mathrm{mRNA} / \beta$-actin mRNA. All primer pairs were obtained from Shenggong Biotech Company (Shanghai, China) and were as follows: Cx43 (5'-AAAGGCGTTAAGGATCGCGTG/GTCATCAGGCCGAGGCCT-3') and $\beta$-actin (5'-GTCACCCACACTGTGCCCATCT/ACAGAGTACTTGCGCTCAGGAG-3').

\section{Statistical analysis}

The positive products of $\mathrm{C} \times 43$ were analyzed with the Motic Image Advanced 3.0 image analysis system. We immunostained the 3 sections in the transplant core and boundary of the $\mathrm{CCl}$ injury with or without transplantation of NSCs per animal $(n=3)$. Under the microscope (400x), 3 regions per section were counted for $\mathrm{C} \times 43$ positive cells, and the average integral optical density (IOD) was determined. The Western blot and RT-PCR products were semi-quantitatively analyzed by Gel-Pro Analyzer Version 4.0 software (Media Cybernetics, Bethesda, MD) [13]. The ratio of $\mathrm{Cx} 43 / \mathrm{GAPDH}$ or $\mathrm{Cx} 43 / \beta$-actin represented the level of protein or mRNA expression [14]. Independent sample $t$-test was performed to detect the difference between mean values for the NSCs-transplanted rats and the control rats at the same time points. The data were expressed as means \pm standard deviation and the difference was considered significant at $p<0.05$.

\section{Results}

\section{Cx43 immunohistochemical staining}

We detected positive products of $\mathrm{Cx} 43$ in brain sections from NSCs-transplanted rats and the control rats at different time points post-transplantation. Representative $\mathrm{C} \times 43$ (Figures $1 \mathrm{~A}-\mathrm{B}$ ) staining was found in the cell membrane, in the cytoplasm adjacent to the cell membrane, and in the processes in injured brain with or without NSC transplantation at 1, 2 and 4 weeks. Our study showed that $\mathrm{Cx} 43$ staining was significantly greater in the NSCs-transplanted rats compared to the control rats at different time points (Figure 1C). 

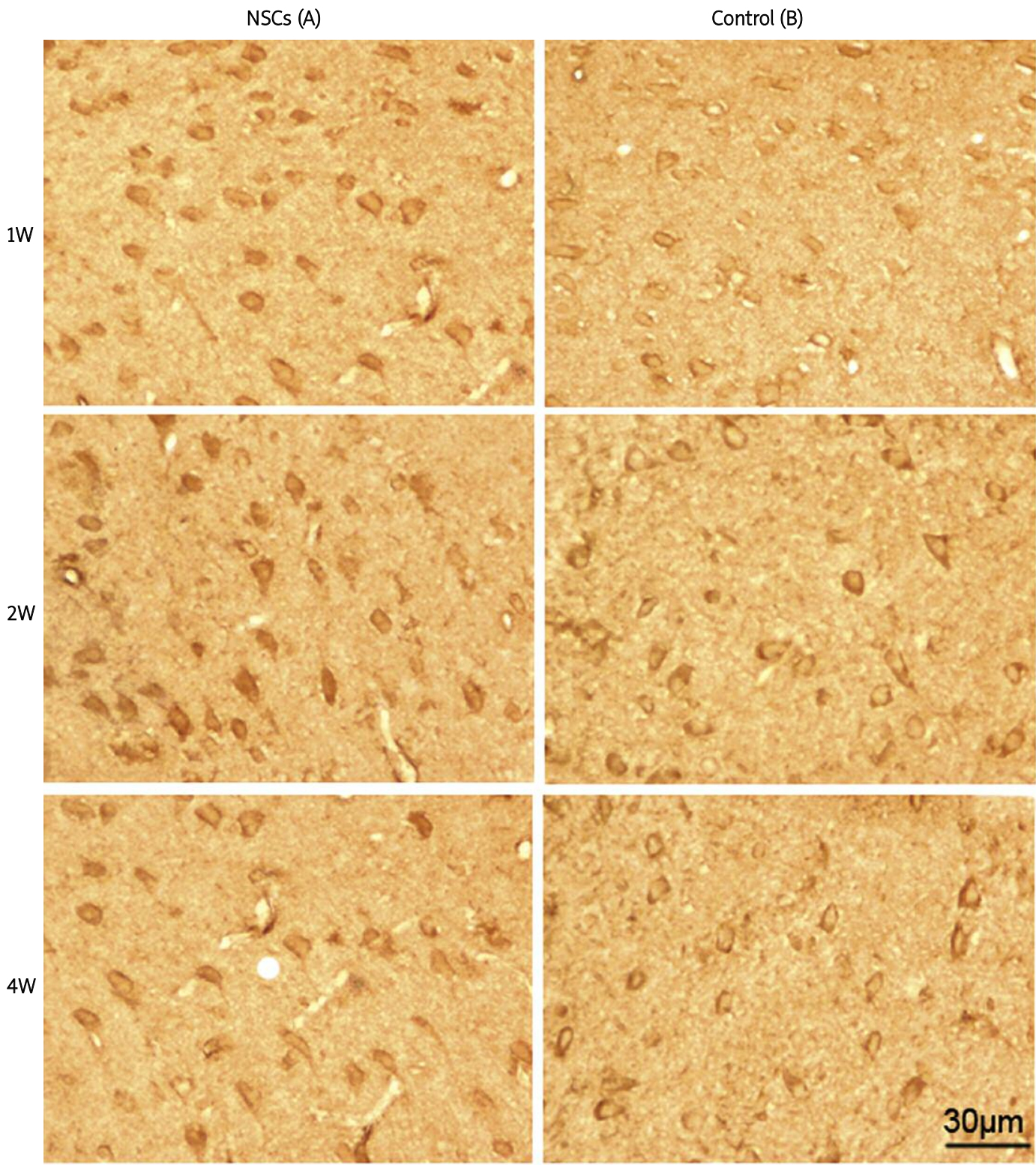

Figure 1. The immunoreactivity of $\mathrm{C} \times 43$ in the transplant core and the border of $\mathrm{CCl}$ at 1, 2 and 4 weeks post-transplantation. (A and B) Cx43-positive staining in $16-\mu \mathrm{m}$ sections from the brains of the NSCs-transplanted and the control rats at 1, 2 and 4 weeks. Immunoreactivity of $\mathrm{C} 43$ was significantly increased in the brains of the NSCs-transplanted rats compared to the control rats at different time points (C). The scale bar represents $30 \mu \mathrm{m}$

${ }^{*}$ and ${ }^{* *}$ indicate $p<0.05$ and $p<0.01$ vs. the control group at different time points

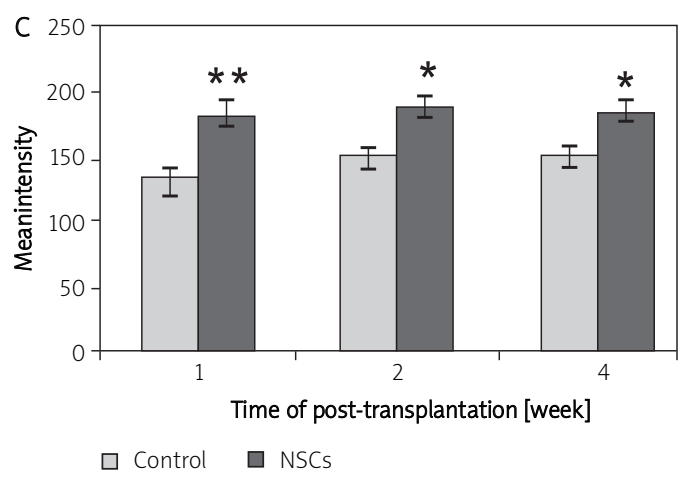

\section{Expression of $\mathrm{C} x 43$ protein and mRNA}

We also performed Western blots and RT-PCR to detect protein and gene expression of $\mathrm{C} \times 43$ in the transplant core and border of the $\mathrm{CCl}$ injury at va-

rious time points post-transplantation. Figure 2 shows the expression of $\mathrm{C} \times 43$ at both the gene (Figures 2 A, B) and protein level (Figures 2 C, D) in the NSCs-transplanted groups and the control groups at various time points post-transplantation. 

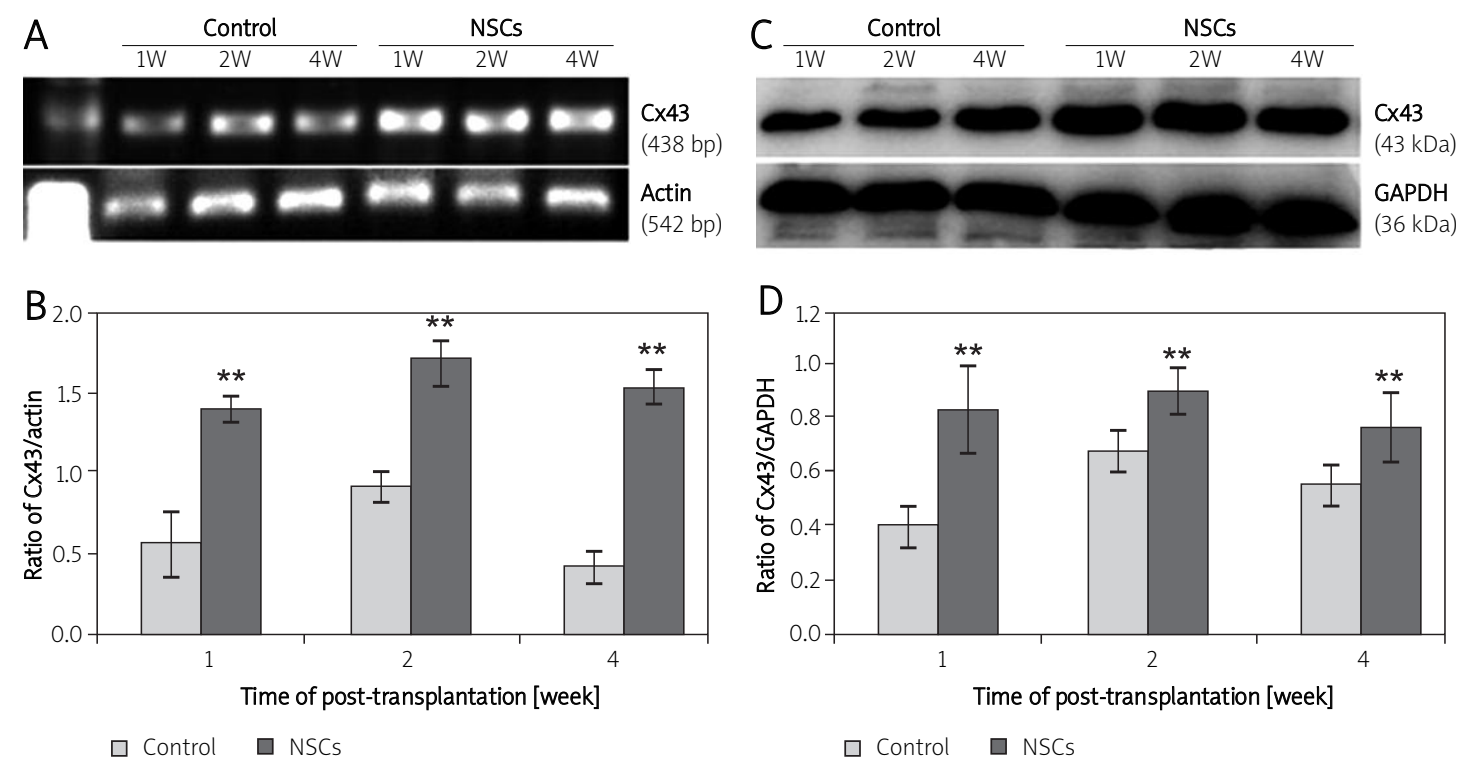

Figure 2. Gene and protein expression of $\mathrm{Cx} 43$ in the injured brain. In (A) and (C), lanes 1,2 and 3 are from control rats and lanes 4, 5 and 6 are from NSCs-transplanted rats. Lanes 1 and 4, 2 and 5, and 3 and 6 are derived from rats at 1,2 and 4 weeks post-transplantation, respectively. Representative results from semi-quantitative RT-PCR for Cx43 (A) and densitometric analysis of these bands (B). The intensity of each band was standardized to the band intensity of $\beta$-actin. Representative Western blots for Cx43 (C) and densitometric analysis of protein bands (D). The intensity of each band was standardized to the band intensity of GAPDH. Data are expressed as the mean \pm SD ( $n=3$ in each group)

${ }^{*}$ and ${ }^{* *}$ indicate $p<0.05$ and $p<0.01$ vs. the control group at different time points

As shown in Figure 2, the PCR product of $\mathrm{Cx} 43$ mRNA was $438 \mathrm{bp}$. The gene expression of $\mathrm{Cx} 43$ was 1.38 -fold, 1.69 -fold, and 1.53-fold at 1, 2, and 4 weeks, respectively, after transplantation in NSCstransplanted rats, and 0.55-fold, 0.91-fold, and 0.42 -fold, respectively, in the control rats (Figures $2 \mathrm{~A}$, $B)$. Significantly higher gene expression of $\mathrm{C} \times 43$ was found in NSCs-transplanted rats compared to the control rats in the period of 4 weeks post-transplantation $(p<0.01)$, and remained at a higher level until 2 weeks with or without NSC transplantation. $\mathrm{C} \times 43$ protein in the transplant core and border of the $\mathrm{CCl}$ injury predominantly migrated as a $43 \mathrm{kDa}$ band as detected by Western blot. The results of the protein and gene expression of $\mathrm{C} \times 43$ were in agreement (Figures 2 C, D).

\section{Discussion}

Our previous study indicated that NSCs were capable of surviving and differentiating and significantly improved neurological function in a rat model of TBI. In this study, we performed the same model of TBI and cell transplantation strategy. Our data showed that NSC transplantation significantly increased the gene and protein expression of gap junction-associated $\mathrm{Cx} 43$ in the transplant core and border of the CCI injury in the NSCs-transplanted rats compared to the saline control rats at 1, 2 and 4 weeks post-transplantation. Enhanced gap junction-associated Cx43 might participate in the NSCs' beneficial effects in mild TBI.
The role of $\mathrm{Cx} 43$ gap junctions and hemichannels in CNS injury can be conceived to be either detrimental or beneficial. A process known as the "good Samaritan" was put forward by Shenton, who proposed a number of possible mechanisms by which gap junction communication might be beneficial in vivo following injury [15]. First of all, cellular injury results in accumulation of deleterious metabolites which are key players in excitotoxicity, such as calcium ions and glutamate in the cytoplasm. Although gap junction communication can also harm healthy neighboring cells by distribution of these toxic materials, a process known as the "bystander effect", gap junction communication promotes cell survival by allowing passage of toxic molecules out of the injured cell and neuroprotective substances, including neurotrophic factors and cytokines secreted by astrocytes, into the cell. In mild injury, increased Cx43 expression might be involved in diffusion or spreading of these deleterious substances, either buffered through gap junctions by healthy cells or released into the extracellular matrix via hemichannels [16], which might otherwise reach toxic levels. Thus, injured cells may be prevented from dying. On the other hand, astrocytes may mediate protection by buffering neurons from excitotoxicity and hypoxic depolarization by taking up extracellular glutamate and potassium, which might stimulate the survival of neighboring neurons and protect neurons from excitotoxic and oxidative insults [17]. Therefore, increased Cx43 is neuroprotective in mild injury. There is some evi- 
dence of increased neurodestruction following global gap junction blockade in vivo. Cx43 null mice exhibited continuous neurodestruction or lesion spread $[18,19]$. In addition, Cx43 might act directly at a genetic level, independent of gap junction or hemichannel activity [20].

Exogenous NSCs can protect or rescue endogenous neurons at risk for degeneration. Apart from diffusible factors that have typically been implicated [21, 22], an important feature that has been recognized is direct contact of NSCs with host cells. Connexin-associated gap junction formation and function are pivotal for ensuring host cell wellbeing. In vivo, engrafted NSCs-mediated rescue of imperiled host neurons did not occur when gap junction formation was suppressed by inhibiting gap junction function. It has been reported that functional gap junctions between grafted NSCs and host cells could be established within 2-18 h after NSCs grafting. Gap-junctional intercellular networks are an early form of communication that precedes and sets the stage for later electrochemical synapses and "traditional" electrophysiological communication between grafted and host cells. It is the earlier connexin, $\mathrm{Cx} 43$, that seems most pivotal in the NSCs-mediated rescue actions [7, 23].

It has also been reported that the role of $\mathrm{C} \times 43$ appears to vary with time following CNS injury. Cx43 mRNA and protein were up-regulated within hours in gray matter rostral to the lesion and reached over 3 times normal levels at 4 weeks following rodent spinal cord transaction injury [24]. Another study found that the injured cortex showed an initial reduction in Cx43-ir up to $6 \mathrm{~h}$ after injury, followed by a subsequent increase in Cx43-ir from $24 \mathrm{~h}$ to $72 \mathrm{~h}$ in the model of TBI compared to controls [25]. It was proposed that it was a critical time in which gap junction coupling and hemichannel activation resulted in the mediation of injury. Initially after a CNS insult Cx43 hemichannels may be involved in the swelling of cells and spread of neurotoxic molecules from injured cells into the extracellular milieu. At later stages spatial buffering through the action of astrocytic gap junctions appears to be required for long-term neuroprotection [15]. What is more important, immunohistochemical staining and dye-transfer studies revealed that the NSCS already formed functional gap junctions prior to engraftment, thereby creating a substrate for subsequent graft and host communication. In an organotypic ex vivo model system for striatal tissue, at 0 and $7 \mathrm{~d}$ 74-94\% of grafted NSCs and NSCs-derived cells expressed Cx43, suggesting a window of opportunity for successful host cell rescue by NSC transplantation [26].

In the present study, we investigated the expression patterns of $\mathrm{Cx} 43$ genes and proteins in the transplant core and the border of $\mathrm{CCl}$, which is regarded as an important site as a therapeutic target because delayed responses take place mainly in the penumbra, and the prevention of delayed responses may be required for successful treatment of injured brain [27]. Our results showed that the gene and protein expression of Cx43 were significantly increased at 1, 2 and 4 weeks post-transplantation compared to the control. We assumed that enhanced NSCs-mediated Cx43 may contribute to NSCs' beneficial effects via gap-junctional coupling in TBI rats treated with NSC transplantation. The mechanisms underlying how gap-junctional coupling facilities NSCs' beneficial effects in vivo require further study. It is likely that initial NSCastrocyte interactions and subsequent NSC-neuron couplings could be important especially during early stages in donor-host interactions. Further functional investigation of gap junction expression in graft and host cells and the associated variations in intercellular communication may help to understand and control the "good Samaritan" effects and "bystander effect" evidently mediated by gap junctional coupling following NSC transplantation after injury and thereby develop a novel therapeutic strategy to alleviate neuronal damage and/or to promote neuronal regeneration in future clinical NSC transplantation therapies.

In conclusion, the gene and protein expression of $\mathrm{Cx} 43$ in the transplant core and the border of $\mathrm{CCl}$ were significantly increased at 1, 2 and 4 weeks following NSC transplantation compared to the control. We assume that enhanced NSCs-mediated Cx43 might contribute to NSCs' beneficial effects via gap-junctional coupling in TBI rats treated with NSC transplantation. Further functional investigation of gap junction expression in exogenous and host cells and the associated changes in intercellular communication may help to understand and control the "good Samaritan" effects and "bystander effect" mediated by gap junctional coupling and thereby develop a novel therapeutic strategy to alleviate neuronal damage and/or to improve neuronal regeneration in future clinical NSC transplantation therapies.

\section{Acknowledgments}

Bo Yu and Haiying Ma contributed equally to this study.

We thank Professor Xuehu Ma and Associate Professor Shui Guan from the Stem Cell and Tissue Engineering Laboratory of the Dalian University of Technology for their support in culturing the NSCs. This work was supported by grants No. 30850001 from the National Nature Science Foundation of China and Nos. 20072167, 2008779 and 2008851 from the S\&T Research Project of Education Bureau, Liaoning Province, China.

\section{References \\ 1. Uyanikgil Y, Balcioglu HA. Neural stem cell therapy in neu- rological diseases. Arch Med Sci 2009; 5: 296-302.}


2. Ma H, Yu B, Kong L, Zhang Y, Shi Y. Transplantation of neural stem cells enhances expression of synaptic protein and promotes functional recovery in a rat model of traumatic brain injury. Mol Med Report 2011; 4: 849-56.

3. Benninger Y, Marino S, Hardegger R, Weissmann C, Aguzzi A, Brandner S. Differentiation and histological analysis of embryonic stem cell-derived neural transplants in mice. Brain Pathol 2000; 10: 330-41.

4. Harting MT, Sloan LE, Jimenez F, Baumgartner J, Cox CS Jr. Subacute neural stem cell therapy for traumatic brain injury. J Surg Res 2009; 153: 188-94.

5. Perez Velazquez JL, Frantseva MV, Naus CC. Gap junctions and neuronal injury: protectants or executioners? Neuro scientist 2003; 9: 5-9.

6. Frantseva MV, Kokarovtseva L, Naus CG, Carlen PL, MacFabe D, Perez Velazquez JL. Specific gap junctions enhance the neuronal vulnerability to brain traumatic injury. J Neu rosci 2002; 22: 644-53.

7. Jäderstad J, Jäderstad LM, Li J, et al. Communication via gap junctions underlies early functional and beneficial interactions between grafted neural stem cells and the host. Proc Natl Acad Sci U S A 2010; 107: 5184-9.

8. Moon Y, Choi SY, Kim K, Kim H, Sun W. Expression of connexin2 2 and 32 in the penumbra region after traumatic brain injury of mice. Neuroreport 2010; 21: 1135-9.

9. Ma XH, Shi Y, Hou Y, et al. Slow-freezing cryopreservation of neural stem cell spheres with different diameters. Cryobiology 2010; 60: 184-91.

10. Yu B, Ma H, Du Z, et al. Involvement of calmodulin and actin in directed differentiation of rat cortical neural stem cells into neurons. Int J Mol Med 2011; 28: 739-44.

11. Carvey PM, Ling ZD, Sortwell CE, et al. A clonal line of mesencephalic progenitor cells converted to dopamine neurons by hematopoietic cytokines: a source of cells for trans plantation in Parkinson's disease. Exp Neurol 2001; 171: 98-108.

12. Bermpohl D, You Z, Korsmeyer SJ, Moskowitz MA, Whalen MJ. Traumatic brain injury in mice deficient in Bid: effects on histopathology and functional outcome. J Cereb Blood Flow Metab 2006; 26: 625-33.

13. Hayashi A, Moradzadeh A, Tong A, et al. Treatment modality affects allograft-derived Schwann cell phenotype and myelinating capacity. Exp Neurol 2008; 212: 324-36.

14. Yu M, Wen N, Wenzhong Z, Yuanchang X, Xiaoming D, Yongjin L. Effect of repeated ischaemic preconditioning on TLR4 and proinflammatory cytokines TNF-alpha and IL-1beta in myocardial ischaemia-reperfusion injury in a rat model. Arch Med Sci 2010; 6: 843-7.

15. Chew SS, Johnson CS, Green CR, Danesh-Meyer HV. Role of connexin43 in central nervous system injury. Exp Neurol 2010; 225: 250-61

16. Ye ZC, Wyeth MS, Baltan-Tekkok S, Ransom BR. Functional hemichannels in astrocytes: a novel mechanism of glutamate release. J Neurosci 2003; 23: 3588-96.

17. Farahani R, Pina-Benabou MH, Kyrozis A, et al. Alterations in metabolism and gap junction expression may determine the role of astrocytes as "good samaritans" or executioners. Glia 2005; 50: 351-61

18. Siushansian R, Bechberger JF, Cechetto DF, Hachinski VC, Naus CC. Connexin43 null mutation increases infarct size after stroke. J Comp Neurol 2001; 440: 387-94.

19. Nakase T, Söhl G, Theis M, Willecke K, Naus CC. Increased apoptosis and inflammation after focal brain ischemia in mice lacking connexin43 in astrocytes. Am J Pathol 2004; 164: 2067-75

20. Dang X, Doble BW, Kardami E. The carboxy-tail of connexin-43 localizes to the nucleus and inhibits cell growth. Mol Cell Biochem 2003; 242: 35-8.
21. Li J, Imitola J, Snyder EY, Sidman RL. Neural stem cells res cue nervous purkinje neurons by restoring molecular homeostasis of tissue plasminogen activator and downstream targets. J Neurosci 2006; 26: 7839-48.

22. Redmond DE Jr, Bjugstad KB, Teng YD, et al. Behavioral improvement in a primate Parkinson's model is associated with multiple homeostatic effects of human neural stem cells. Proc Natl Acad Sci U S A 2007; 104: 12175-80.

23. Jäderstad LM, Jäderstad J, Herlenius E. Graft and host interactions following transplantation of neural stem cells to organotypic striatal cultures. Regen Med 2010; 5: 901-17.

24. Lee IH, Lindqvist E, Kiehn O, Widenfalk J, Olson L. Glial and neuronal connexin expression patterns in the rat spinal cord during development and following injury. J Comp Neurol 2005; 489: 1-10.

25. Ohsumi A, Nawashiro $\mathrm{H}$, Otani N, et al. Alteration of gap junction proteins (connexins) following lateral fluid percussion injury in rats. Acta Neurochir Suppl 2006; 96: 148-50.

26. Jäderstad J, Jäderstad LM, Herlenius E. Dynamic changes in connexin expression following engraftment of neural stem cells to striatal tissue. Exp Cell Res 2010; 317: 70-81.

27. Paciaroni M, Caso V, Agnelli G. The concept of ischemic penumbra in acute stroke and therapeutic opportunities. Eur Neurol 2009; 61: 321-30. 